\title{
Cardiothoracic
}

Transplantation

\section{Primary graft dysfunction and other selected complications of lung transplantation: A single-center experience of 983 patients}

\author{
Bryan F. Meyers, MD, ${ }^{a}$ Maite de la Morena, MD, ${ }^{c}$ Stuart C. Sweet, MD, ${ }^{c}$ Elbert P. Trulock, MD, ${ }^{b}$ \\ Tracey J. Guthrie, RN, BSN, ${ }^{a}$ Eric N. Mendeloff, MD, ${ }^{a}$ Charles Huddleston, MD, ${ }^{a}$ Joel D. Cooper, MD, and \\ G. Alexander Patterson, $\mathrm{MD}^{\mathrm{a}}$
}

Figures are available online. $\mathcal{B}$
From the Division of Cardiothoracic Surgery, Department of Surgery; the Division of Pulmonary Medicine and Critical Care, Department of Internal Medicine; the Department of Pediatrics; Washington University School of Medicine, St Louis, Mo, and the Jacqueline Maritz Lung Center ${ }^{\mathrm{a}, \mathrm{b}}$ at Barnes-Jewish Hospital, St Louis Children's Hospital, ${ }^{\mathrm{c}}$ St Louis, Mo.

Read at the Eighty-fourth Annual Meeting of The American Association for Thoracic Surgery, Toronto, Ontario, Canada, April 25-28, 2004.

Received for publication April 22, 2004; revisions received Dec 15, 2004; accepted for publication Jan 13, 2005.

Address for reprints: Bryan F. Meyers, MD, One Barnes-Jewish Plaza, 3108 Queeny Tower, St Louis, MO 63110 (E-mail: meyersb@wustl.edu).

J Thorac Cardiovasc Surg 2005;129:1421-9

$0022-5223 / \$ 30.00$

Copyright (c) 2005 by The American Association for Thoracic Surgery

doi:10.1016/j.jtcvs.2005.01.022
Objectives: We sought to review the incidence and outcome of lung transplantation complications observed over 15 years at a single center.

Methods: We performed a retrospective review from our databases, tracking outcomes after adult and pediatric lung transplantation. The 983 operations between July 1988 and September 2003 included 277 pediatric and 706 adult recipients. Bilateral (74\%), unilateral (19\%), and living lobar transplants (4\%) comprised the bulk of this experience. Retransplantations accounted for $44(4.5 \%)$ of the operations.

Results: The groups differed by indication for transplantation. The adults included $57 \%$ with emphysema and $17 \%$ with cystic fibrosis, and the children included no patients with emphysema and $50 \%$ with cystic fibrosis. Hospital mortality was $96(9.8 \%)$ of 983 , including $46(17 \%)$ of 277 of the children and $50(7 \%)$ of 706 of the adults. The overall survival curves did not differ between adults and children $(P=.56)$. Freedom from bronchiolitis obliterans syndrome at 5 and 10 years was $45 \%$ and $18 \%$ for adults and $48 \%$ and $30 \%$ for children, respectively $(P=.53)$. The causes of death for adults included bronchiolitis obliterans syndrome $(40 \%)$, respiratory failure $(17 \%)$, and infection $(14 \%)$, whereas the causes of death in children included bronchiolitis obliterans syndrome $(35 \%)$, infection $(28 \%)$, and respiratory failure $(21 \%)(P<.01)$. Posttransplantation lymphoproliferative disease occurred in $12 \%$ of pediatric recipients and $6 \%$ of adults $(P<.01)$. The frequency of treated airway complications did not differ between adults and children $(9 \%$ vs $11 \%, P=.48)$. The frequency of primary graft dysfunction did not differ between children $(22 \%)$ and adults $(23 \%)$, despite disparity in the use of cardiopulmonary bypass.

Conclusion: These results highlight major complications after lung transplantation. Despite differences in underlying diagnoses and operative techniques, the 2 cohorts of patients experienced remarkably similar outcomes.

$\mathrm{L}$ ung transplantation has been transformed from extraordinary therapy in the early 1980s to widely available therapy in the current era. The transformation has been marked by a change in recipient selection, from those who were facing certain death with no options in the early 1980s to patients looking to increase both life expectancy and functional status in the current era. Attitudes concerning 
TABLE 1. Recipients stratified by pediatric versus adult classification

\begin{tabular}{|c|c|c|c|}
\hline & Pediatric (n = 277) & Adult $(n=706)$ & $P$ value \\
\hline Age (y) & $10.7 \pm 6.4 ; 12.1$ (IOR, 5.4-15.8) & $47.6 \pm 12.2 ; 50.5(\operatorname{IQR}, 38.9-57.6)$ & \\
\hline $\operatorname{Sex}(F)$ & $156(56.3 \%)$ & $357(50.6 \%)$ & .118 \\
\hline Diagnosis & & & $<.001$ \\
\hline Emphysema & $0(0 \%)$ & $399(56.5 \%)$ & \\
\hline Cystic fibrosis & $138(49.8 \%)$ & $118(16.7 \%)$ & \\
\hline Pulmonary vascular disease & $71(25.6 \%)$ & $63(8.9 \%)$ & \\
\hline Other pulmonary disease & $68(24.6 \%)$ & $126(17.8 \%)$ & \\
\hline Time on the waiting list (d) & 176 (IOR,42-465) & 488 (IOR,281-665) & $<.001$ \\
\hline Type of transplantation performed & & & $<.001$ \\
\hline Bilateral & $204(73.6 \%)$ & $526(74.5 \%)$ & \\
\hline Single & $8(2.9 \%)$ & $176(24.9 \%)$ & \\
\hline Heart-lung & $16(5.8 \%)$ & $4(0.6 \%)$ & \\
\hline Bilateral lobar (living) & $39(14.1 \%)$ & $0(0 \%)$ & \\
\hline Bilateral lobar (cadaveric) & $4(1.4 \%)$ & $0(0 \%)$ & \\
\hline Single lobe & $1(0.4 \%)$ & $0(0 \%)$ & \\
\hline Bilateral-liver & $5(1.8 \%)$ & $0(0 \%)$ & \\
\hline Length of mechanical ventilation (d) & $5(I 0 R, 2-14)$ & $2(I O R, 1-4)$ & $<.001$ \\
\hline Length of stay in ICU (d) & 7 (IOR, 4-17) & $3(\operatorname{IQR}, 2-5)$ & $<.001$ \\
\hline Length of stay in hospital (d) & 19 (IOR, 13-33) & 17 (IOR, 12-24) & $<.001$ \\
\hline $\begin{array}{l}\text { Required ECMO support after } \\
\text { transplantation }\end{array}$ & $27(9.7 \%)$ & $20(2.8 \%)$ & $<.001$ \\
\hline Primary graft dysfunction* & $62(22.4 \%)$ & $160(22.7 \%)$ & 1.000 \\
\hline Hospital mortality & $46(16.6 \%)$ & $50(7.1 \%)$ & $<.001$ \\
\hline Treated for airway complication & $30(10.8 \%)$ & $66(9.3 \%)$ & .476 \\
\hline $\begin{array}{l}\text { Diagnosed with posttransplantation } \\
\text { lymphoproliferative disease }\end{array}$ & $32(11.6 \%)$ & $42(5.9 \%)$ & .004 \\
\hline
\end{tabular}

IQR, Interquartile range; ICU, intensive care unit; ECMO, extracorporeal membrane oxygenation. *Adults: arterial partial pressure of oxygen/fraction of inspired oxygen of 150 or less on initial or 24-hour arterial blood gas; Peds: mechanical ventilation $>7$ days, retransplantation $<7$ days of initial transplant, chest radiograph consistent with reperfusion injury, biopsy.

lung transplantation outcomes have gradually shifted as well, from the initial amazement that it could be done successfully at all to increasing pressure to reduce morbidity and mortality while at the same time justifying the expense with cost-effectiveness analyses. Both the effectiveness and the cost of lung transplantation are greatly affected by complications. We chose to review important selected complications of our overall lung transplantation program from the time of its inception to highlight important differences between the adult and pediatric programs and to identify trends in the incidence and severity of our complications over time. The goal is to provide increased awareness of such trends and to discuss how they might affect future work in the field of lung transplantation.

\section{Methods}

This report is a single-center retrospective report that examines complications after lung transplantation in adults and children. The lung transplantation program at Washington University is composed of 2 independent programs. The adult lung transplantation effort opened in 1988, and the pediatric program began in 1990. Since the start of the program at Children's Hospital, the 2 programs have been managed separately, with distinct sur- geons and pulmonologists at each institution. There has been close interchange of results and sharing of innovation. The databases of the 2 programs were used jointly in this project to provide a single-center look at lung transplantation since the onset of the 2 programs. The databases for the 2 programs were queried to obtain data regarding the indications for and outcomes of all lung transplantations performed since 1988 at $\mathrm{St}$ Louis Children's Hospital and Barnes-Jewish Hospital. Individual patient records were consulted only to verify missing, incomplete, or suspect data.

\section{Patient Population}

The patients in this report include all patients undergoing lung transplantation or heart-lung transplantation at Barnes-Jewish Hospital or St Louis Children's Hospital from July 1988 through September 2003. Table 1 describes the demographic data of these patients.

\section{Surgical Technique}

The techniques have been well described elsewhere. ${ }^{1,2}$ In general, the techniques have been consistent over time and between the 2 programs, with a few notable exceptions. Although both programs prefer the bilateral lung transplantation option, the pediatric group has performed some form of bilateral transplantation in 268 (97\%) of 277 operations, whereas the adult experience includes bilateral transplan- 
tation in $530(75 \%)$ of 706 procedures. Virtually all of the pediatric operations were performed with cardiopulmonary bypass because of the relative difficulties of double-lumen endotracheal tube use in children and the prevalence of pulmonary hypertension in the pediatric cohort. On the other hand, the rate of bypass support in the adult population varied greatly according to diagnosis and the presence of pulmonary hypertension.

\section{Postoperative Management}

Routine postoperative management has been discussed elsewhere. $^{3,4}$ In general, the management has been consistent over time and between the 2 programs. Both programs have depended on 3-drug immunosuppression (typically prednisone, cyclosporine [INN: ciclosporin], and azathioprine), and both programs have routine scheduled surveillance bronchoscopy to guide the maintenance immunosuppression.

\section{Statistical Analysis}

Descriptive statistics were expressed as means \pm standard deviation unless otherwise specified. Categoric data were expressed as counts and proportions. Comparisons were done with paired, 2-tailed $t$ tests for means of normally distributed continuous variables and Wilcoxon rank sum tests for skewed data. Fisher exact or $\chi^{2}$ tests were used to analyze differences among the categorical data. Kaplan-Meier estimate was used to depict survival. All data analysis was performed with SPSS software (SPSS 11.0 for Windows; SPSS Inc, Chicago, Ill).

\section{Results}

During the period of reporting, there were 983 lung transplantations, including 277 performed at St Louis Children's Hospital and 706 at Barnes-Jewish Hospital. Although these might roughly be partitioned as pediatric and adult transplantations, there was some small overlap with regard to age (Figure E1). Overall hospital mortality was 96 (9.8\%) of 983 and included 46 (17\%) of 277 in the pediatric group and $50(7 \%)$ of 706 in the adult group. The typical waiting time for donor lungs varied according to transplantation site. There was a statistically significant difference in the distribution of recipient diagnoses, with the notable predominance of cystic fibrosis as a leading diagnosis for the children and emphysema as the most common indication for transplantation in the adults. In both locations the split according to sex was approximately equal. There were statistically significant and clinically meaningful differences in the duration of mechanical ventilation and duration of intensive care unit (ICU) stay after lung transplantation. The children remained intubated for a median of 5 days (interquartile range, 2-14), whereas the adults experienced a median length of mechanical ventilation of 2 days (interquartile range, 1-4 days).

\section{Primary Graft Dysfunction}

One of the most important complications of lung transplantation is primary graft dysfunction (PGD). This term includes a wide variety of other definitions, including ischemia-reperfusion injury, implantation response, acute lung injury, and hyperacute rejection. Although the coding of the databases at St Louis Children's Hospital and Barnes-Jewish Hospital have slightly different criteria for coding a case as "yes" for PGD, the incidence has been consistent throughout the experience and is comparable with that seen in multiple reports from other programs in the literature. At Barnes-Jewish Hospital, the criteria for PGD are based on the ratio of $\mathrm{PaO}_{2}$ divided by the fraction of inspired oxygen in the first 48 hours after transplantation. Cases were defined as those having a $\mathrm{PaO}_{2} /$ fraction of inspired oxygen ratio of less than 150 $\mathrm{mm} \mathrm{Hg}$ in the first 48 hours. At Children's Hospital, the coding was performed by one of the authors (S.C.S.), who identified cases as those demonstrating the presence of (1) mechanical ventilation beyond 7 days after transplantation or experiencing retransplantation before 7 days and (2) either chest radiographs showing panlobar alveolar infiltrates or lung biopsy diagnosis of diffuse alveolar damage or reperfusion injury. We identified 62 (22.4\%) of 277 pediatric cases and 160 (22.7\%) of 706 adult cases of PGD in this manner. The incidence of PGD varied slightly over time, but the differences were not larger than one might expect from chance alone (Table 2 ). There was a statistically significant difference in the distribution of PGD according to the underlying diagnosis leading to transplantation. There was an apparent excess of PGD in the patients who underwent transplantation for pulmonary hypertension and an apparent sparing of patients who underwent transplantation for emphysema.

The effect of PGD on the transplantation program as a whole is enormous. The mortality of patients with PGD was $64(28.8 \%)$ of 222 compared with $32(4.2 \%)$ of 761 seen in those patients without the condition $(P<.001)$. The risk of death for the entire cohort of 983 patients that is attributable to PGD can be estimated by the risk in the total cohort $(96 / 983$ [9.8\%]) minus the risk in the unexposed group (32/761 [4.2\%]). Therefore the phenomenon of PGD might be responsible for a mortality of $5.6 \%$ by itself, more than half of the overall death rate for the entire cohort (Figure 1).

In addition to the burden of excess mortality in patients undergoing transplantation, PGD is also responsible for a substantial increase in the cost of providing this medical care. In these 980 patients, the costs that could be reasonably attributed to PGD can be estimated by multiplying the increased resource use in the afflicted group by the number of patients in that group. For instance, the 222 patients with PGD were intubated for a median of 6 days, whereas those without PGD were intubated for 2 days. The excess time of intubation (4 days) associated with PGD can be multiplied 
TABLE 2. Factors associated with primary graft dysfunction

\begin{tabular}{lcc}
\hline & PGD, no (n = 761) & PGD, yes (n= 222) \\
\hline Quintile of experience & & \\
$\quad 7 / 1988-3 / 1993$ & $144(73.5 \%)$ & $52(26.5 \%)$ \\
$4 / 1993-11 / 1995$ & $154(78.2 \%)$ & $43(21.8 \%)$ \\
$12 / 1995-5 / 1998$ & $156(79.2 \%)$ & $41(20.8 \%)$ \\
$5 / 1998-1 / 2001$ & $161(81.7 \%)$ & $36(18.3 \%)$ \\
$1 / 2001-9 / 2003$ & $146(74.5 \%)$ & $50(25.5 \%)$ \\
Diagnosis & & \\
Emphysema & $325(81.5 \%)$ & $74(18.5 \%)$ \\
Cystic fibrosis & $197(77.0 \%)$ & $59(23.0 \%)$ \\
Pulmonary vascular disease & $94(70.1 \%)$ & $40(29.9 \%)$ \\
Other pulmonary disease & $145(74.7 \%)$ & $49(25.3 \%)$ \\
Ischemic time (min) & $274 \pm 70$ & $290 \pm 86$ \\
Length of mechanical ventilation (d) & $2(I 0 R, 1-4)$ & $6(I 0 R, 2-18)$ \\
Length of stay in ICU (d) & $3(I 0 R, 2-6)$ & $7(I 0 R, 3-18)$ \\
Length of stay in hospital (d) & $16(I 0 R, 12-23)$ & $23(I 0 R, 13-39)$ \\
Hospital mortality & $32(4.2 \%)$ & $64(28.8 \%)$ \\
\hline
\end{tabular}

$P G D$, Primary graft dysfunction; $I Q R$, interquartile range; $I C U$, intensive care unit.

by 222 patients with PGD to come up with 888 patient-days on the ventilator attributable to the condition of PGD. A similar difference in days in the ICU (7 for those with PGD vs 3 for those without PGD) leads to an excess of 888 patient-days in the ICU attributable to PGD. The difference in hospital stay for those with and without PGD is 7 days, and therefore the attributable burden of hospital days is 7 times 222 , or 1554, patient-days. Because 888 of the patient-days are already counted in the ICU stay, this would amount to 1554 minus 888 , or 666 , non-ICU patient-days attributable to PGD.

Another costly consequence of early lung dysfunction is the burden of acute retransplantation. This controversial therapy has been performed 11 times in our collective experience, 5 in pediatric recipients and 6 in adults. In these instances the resources consumed as a direct consequence of PGD are not limited to financial and hospital resources.

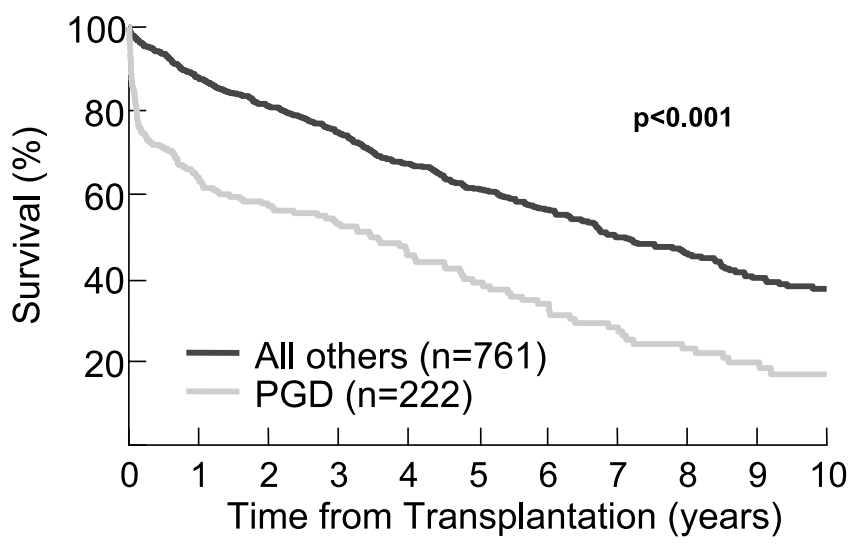

Figure 1. Survival after lung transplantation stratified by PGD.
They also include the very limited availability of lung allografts suitable for transplantation. In this manner the use of acute retransplantation as a response to severe PGD has survival implications even for patients on the waiting list because their likelihood of undergoing successful transplantation will decrease as the use of allografts for retransplantation increases.

In addition to the acute effects of PGD, there has been some evidence that this early difficulty increases the recipient's likelihood for chronic graft dysfunction as well. We observed a small but statistically significant increase in the hazard for chronic rejection in the survivors of PGD versus those who did not have the condition. This tendency is shown by the reduction in the mean time to development of bronchiolitis obliterans syndrome (BOS), from 4.5 years (95\% confidence interval $[\mathrm{CI}], 3.8-5.2)$ in those without PGD to 3.3 years $(95 \% \mathrm{CI}, 2.2-4.4)$ in those with PGD. A comparison of freedom from BOS in those with and without PGD revealed a statistically significant association by using the log-rank test $(P=.044$, Figure E2).

One therapeutic intervention that might become necessary in the acute management of early lung dysfunction is extracorporeal membrane oxygenation (ECMO). We have previously reported some outcomes of ECMO support in our adult lung transplantation population and concluded that the best likelihood for survival occurs when ECMO support is instituted within 24 hours of the transplantation and lung recovery allows weaning from ECMO within 48 hours of its initiation. ${ }^{5}$ In this cumulative experience 47 of 983 patients received ECMO support after the transplantation for the constellation of findings consistent with PGD: poor oxygenation despite maximal therapeutic interventions, stiff lungs with high airway pressures, and respiratory acidosis. There 
TABLE 3. Factors associated with airway anastomotic complications

\begin{tabular}{|c|c|c|c|}
\hline & $A C$, no $(n=887)$ & AC, yes $(n=96)$ & $P$ value \\
\hline Population & & & .476 \\
\hline Adult & $640(90.7 \%)$ & $66(9.3 \%)$ & \\
\hline Pediatric & $247(89.2 \%)$ & $30(10.8 \%)$ & \\
\hline Quintile & & & .016 \\
\hline 7/1988-3/1993 & $165(84.2 \%)$ & $31(15.8 \%)$ & \\
\hline 4/1993-11/1995 & $178(90.4 \%)$ & $19(9.6 \%)$ & \\
\hline 12/1995-5/1998 & $185(93.9 \%)$ & $12(6.1 \%)$ & \\
\hline $5 / 1998-1 / 2001$ & $182(92.4 \%)$ & $15(7.6 \%)$ & \\
\hline $1 / 2001-9 / 2003$ & $177(90.3 \%)$ & $19(9.7 \%)$ & \\
\hline Diagnosis & & & .718 \\
\hline Emphysema & $364(91.2 \%)$ & $35(8.8 \%)$ & \\
\hline Cystic fibrosis & $232(90.6 \%)$ & $24(9.4 \%)$ & \\
\hline Pulmonary vascular disease & $119(88.8 \%)$ & $15(11.2 \%)$ & \\
\hline Other pulmonary disease & $172(88.7 \%)$ & $22(11.3 \%)$ & \\
\hline Primary graft dysfunction & $197(22.2 \%)$ & $25(26.0 \%)$ & .440 \\
\hline Hospital mortality & $86(9.7 \%)$ & $10(10.4 \%)$ & .856 \\
\hline
\end{tabular}

$A C$, Anastomotic complications.

was an association between the classification of pediatric or adult recipient and the use of ECMO, with 27 (9.7\%) of 277 pediatric patients requiring ECMO, whereas $20(2.8 \%)$ of 706 adult patients were started on ECMO postoperatively. The mortality among the ECMO recipients was $29(62 \%)$ of 47 , with a relative risk of death of 8.6 (95\% CI, 6.3-11.9) compared with the non-ECMO recipients.

\section{Airway Anastomotic Complications}

One of the sentinel complications in lung transplantation is the development of an airway anastomotic complication that requires therapy. ${ }^{6}$ In our overall cohort there was no association between the rate of airway complications and classification of adult versus pediatric transplantation. The adults experienced a rate of anastomotic complication of 66 (9.3\%) of 706 , whereas the pediatric airway complication rate was $30(10.8 \%)$ of 277 . In review of the entire experience over time, there was an association between the rate and quintile of experience $(P=.016)$, with the highest rate occurring in the initial quintile of experience from 1988 through 1993 (Table 3). There was no association between patient diagnosis and the rate of anastomotic complications $(P=.72)$. Analysis to determine the effect of acute graft dysfunction on the development of airway complications failed to show a statistically significant association between these outcomes. The odds ratio describing the association between PGD and subsequent airway complications is 1.2 (95\% CI, 0.8-1.85).

Although airway complications appear to affect about $10 \%$ of our transplant recipients and might lead to increased costs and decreased quality of life, the presence of an airway complication does not appear to be associated with survival. The hospital mortality of those with an airway complication was $10(10 \%)$ of 96 versus a mortality for those without airway complications of 86 (9.7\%) of 887 . The log-rank test comparing overall survival in the 2 groups showed no association between airway complication and survival $(P=.76)$. The median survival of those with an airway complication was 6.9 years (95\% CI, 4.1-9.6), whereas the median survival of those without airway complications was 6.1 years $(95 \%$ CI, 2.8-6.2). This comparison might be biased by the fact that very early mortality would prevent a patient from being alive long enough to be at risk for a symptomatic and treatable airway complication.

\section{BOS and Posttransplantation Lymphoproliferative Disease}

Although PGD is the dominant problem faced early after lung transplantation, the development of chronic rejection or BOS is the major driver of morbidity in the late posttransplantation period. Figure E3 shows that there is no difference between the adult and the pediatric experience with regard to freedom from BOS over time. The median time to the diagnosis of BOS is 4.5 years (95\% CI, 2.8-6.2) for the pediatric group and 4.3 years $(95 \% \mathrm{CI}, 3.6-4.9)$ for the adult population.

The frequency with which posttransplantation lymphoproliferative disease (PTLD) was diagnosed in the posttransplantation period is $5.9 \%$ of the adults and $11.6 \%$ of the children $(P=.004)$. We do not have full data on pretransplantation Epstein-Barr virus status and cannot provide further analysis with regard to risk factors for the development of this serious complication.

Long-term survival is also comparable between the groups. The 10-year survival curves are depicted in Fig- 


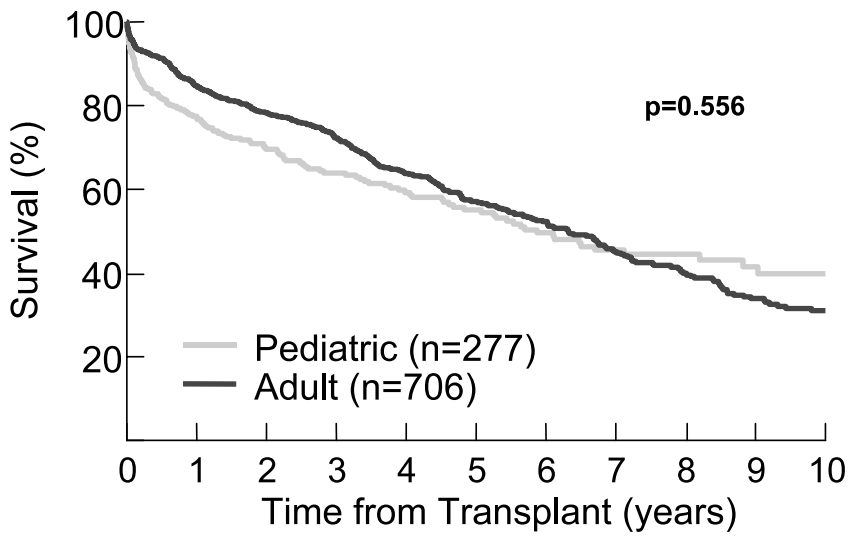

Figure 2. Survival after ling transplantation stratified by patient population.

ure 2. There is no significant difference between the survival curves $(P=.56)$, and the median time to death is 5.9 years $(95 \% \mathrm{CI}, 4.4-7.4)$ for the pediatric cohort and 6.3 years (95\% CI, 5.6-7.0) for the adults. The causes of death are listed in Figure 3, with BOS and graft failure together accounting for the majority of the deaths.

\section{Discussion}

This article provides a single-center experience of the complications of lung transplantation over a 15 -year period. The major limitation of such a report is the retrospective nature of the work. The lung transplantation databases for the 2 hospitals described were not up and running at the time of the first transplantation, and therefore many of the entries were made after the fact at the time of the creation of the databases. In some instances, the lack of a uniform definition might lead to differential reports of complications. Another potential limitation of a cumulative report such as this is the disparate nature of the 2 experiences. There are no children who underwent transplantation for emphysema, yet that diagnosis makes up the largest fraction of the adult cohort. On the other hand, secondary pulmonary hypertension caused by a congenital heart disorder is an extremely unusual indication for an adult but a common one for an infant. To analyze all of these patients together is an invitation to introduce bias and confounding.

Lung transplantation is a therapy for which the costs and incremental cost-effectiveness ratio are coming under increasing scrutiny. Many analyses have concluded that it is an expensive but effective therapy. A study by the University of Pittsburgh in 1997 reported median costs of $\$ 94,000$ per transplantation and average costs of $\$ 154,000$ per transplantation, a disparity that was due to some higher cost outliers. ${ }^{7}$ A Dutch study in 1998 that assessed incremental cost-effectiveness of transplantation over medical therapy
Pediatric $(\mathrm{n}=277)$

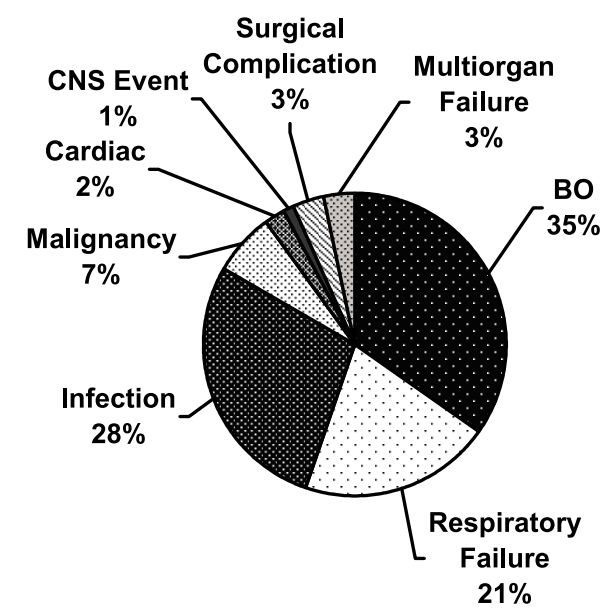

Adult ( $n=706)$

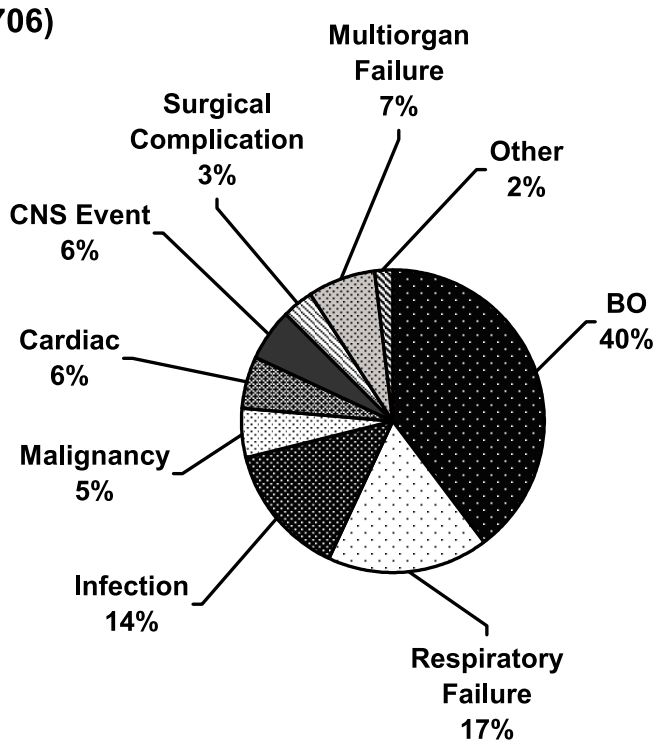

Figure 3. Cause of death stratified by adult versus pediatric status. CNS, Central nervous system; $B O$, bronchiolitis obliterans.

reported a baseline scenario in which the incremental cost per quality-adjusted life year gained was $\$ 116,000$ for lung transplantation compared with medical therapy. ${ }^{8}$ The most recent and comprehensive of such analyses was the UK study that compared the cost-effectiveness of single, bilateral, and heart-lung transplantation with an estimated cost per quality-adjusted life year for these operations of $\$ 48,000, \$ 33,000$, and $\$ 29,000$, respectively. Although the latter estimates are within the range of values that society is readily willing to pay for health care interventions, the other 
previous estimates are perhaps more representative of the situation in the United States. ${ }^{9}$

It is apparent from these data that PGD creates a substantial burden on the population of lung transplant recipients. On the basis of the attributable risks of death, hospital resource consumption, and allograft, a reduction of the rate of PGD by $50 \%$ (from $22 \%$ to $11 \%$ ) in our population could have saved 50 lives, eliminated the need for 6 retransplantations, and reduced hospital resource consumption by more than 400 ICU-ventilator days, as well as more than 300 additional non-ICU days. Our review did not reveal any reliable clinical prediction rules that might identify highrisk recipients or donors, and our rate of PGD appears comparable with rates reported by others. ${ }^{10-13}$

The differences we report in the rates of PTLD in children and adults confirms earlier work by others who have reported rates of 5\% or less in adults and rates as high as $16 \%$ in children. ${ }^{14-17}$ Risk factors for this neoplasm appear to be pretransplant seronegativity for Epstein-Barr virus and lower age. It is typical for reports to describe a $50 \%$ mortality, with the majority of the deaths occurring within a few months of the diagnosis of the PTLD. ${ }^{17-20}$

It is a bit concerning that the trend of any specific complication over time does not show a steady trend toward elimination of that complication. The incidence of PGD seemed to be trending downward, but a recent increase has erased the improvements seen in the midportion of the experience (Table 2). The rate of anastomotic complications decreased rapidly in the early experience and has seen little change in the last decade (Table 3). On the surface, one might hope that experience would bring increased skill and judgment sufficient to gradually eliminate all but the most unpredictable complications. It is of course more complicated than that. One must keep in mind the multiple subtle changes in many aspects of these patients' care that have taken place over the period of observation. In the earliest era, stringent criteria were required before declaring potential donor lungs acceptable for transplantation. Gradually, experience accumulated that demonstrated the safety and effectiveness of using less-than-perfect donor lungs for most recipient indications. Other changes over the years have been subtle evolution of anastomotic techniques, multiple alterations of lung procurement and cold preservation, and intermittent changes to the cohort of surgeons performing the implantations. The growing list of waiting patients makes transplantation teams now willing to accept organs from distant cities with the full knowledge that the increased travel times will prolong the ischemic times and increase the risk for early and late graft dysfunction. In many instances the changes in decision making have been a conscious decision to accept the possibility of a less ideal outcome to increase the supply of donor organs and therefore increase the availability of lung transplantation in general.

\section{Conclusions}

A focus on complications is important because it identifies areas that can be prioritized for future work in reducing costs and improving benefits of lung transplantation. If one considers areas to improve the cost-effectiveness of a therapy, the reduction of complications is a fertile area for progress because those complicated patients have both the highest cost of care and the worst quality and duration of survival. The reduction might be achieved in many ways, ranging from adjustments in technique to eliminate the hazard of complications to adjustment in selection criteria to emphasize potential recipients in whom a safe and effective outcome is most likely to be achieved.

\section{References}

1. Pasque MK, Cooper JD, Kaiser LR, Haydock DA, Triantafillou A, Trulock EP. An improved technique for bilateral lung transplantation: rationale and initial clinical experience. Ann Thorac Surg. 1990;49: 785-91.

2. Huddleston C, Bloch JB, Sweet SC, de la Morena M, Patterson GA, Mendeloff EN. Lung transplantation in children. Ann Surg. 2002;236: 270-6.

3. Sweet S, Spray T, Huddleston C, Mendeloff E, Canter C, Balzer D, et al. Pediatric lung transplantation at St. Louis Children's Hospital. Am J Respir Crit Care Med. 1997;155:1027-35.

4. Trulock EP. Lung transplantation. Am J Respir Crit Care Med. 1997; 155:789-818.

5. Meyers BF, Sundt TM, Henry S, Trulock EP, Guthrie T, Cooper JD, et al. Selective use of extracorporeal membrane oxygenation is warranted after lung transplantation. J Thorac Cardiovasc Surg. 2000;120: 20-8.

6. Date H, Trulock EP, Arcidi JM, Sundaresan S, Cooper JD, Patterson GA. Improved airway healing after lung transplantation: an analysis of 348 bronchial anastomoses. J Thorac Cardiovasc Surg. 1995;110: 1424-33.

7. Gartner SH, Sevick MA, Keenan RJ, Chen GJ. Cost-utility of lung transplantation: a pilot study. J Heart Lung Transplant. 1997;16:112934.

8. Al MJ, Koopmanschap MA, van Enckevort PJ, Geertsma A, van der Bij W, de Boer WJ, et al. Cost-effectiveness of lung transplantation in The Netherlands: a scenario analysis. Chest. 1998;113:124-30.

9. Anyanwu AC, McGuire A, Rogers CA, Murday AJ. An economic evaluation of lung transplantation. J Thorac Cardiovasc Surg. 2002; 123:411-20.

10. Fiser SM, Kron IL, Long SM, Kaza AK, Kern JA, Cassada DC, et al. Influence of graft ischemic time on outcomes following lung transplantation. J Heart Lung Transplant. 2001;20:1291-6.

11. Ardehali A, Laks H, Levine M, Shpiner R, Ross D, Watson LD, et al. A prospective trial of inhaled nitric oxide in clinical lung transplantation. Transplantation. 2001;72:112-5.

12. Meade MO, Granton JT, Matte-Martyn A, McRae K, Weaver B, Cripps $\mathrm{P}$, et al. A randomized trial of inhaled nitric oxide to prevent ischemia-reperfusion injury after lung transplantation. Am J Respir Crit Care Med. 2003;167:1483-9.

13. Thabut G, Brugiere O, Leseche G, Stern JB, Fradj K, Herve P, et al. Preventive effect of inhaled nitric oxide and pentoxifylline on ischemia/reperfusion injury after lung transplantation. Transplantation. 2001;71:1295-300.

14. Lim GY, Newman B, Kurland G, Webber SA. Posttransplantation lymphoproliferative disorder: manifestations in pediatric thoracic organ recipients. Radiology. 2002;222:699-708.

15. Gao SZ, Chaparro SV, Perlroth M, Montoya JG, Miller JL, DiMiceli $\mathrm{S}$, et al. Post-transplantation lymphoproliferative disease in heart and heart-lung transplant recipients: 30 year experience at Stanford University. J Heart Lung Transplant. 2003;22:505-14. 
16. Ramalingam P, Rybicki L, Smith MD, Abrahams NA, Tubbs RR, Pettay J, et al. Posttransplant lymphoproliferative disorders in lung transplant patients: the Cleveland Clinic experience. Mod Pathol. 2002;15:647-56.

17. Reams BD, McAdams HP, Howell DN, Steele MP, Davis RD, Palmer SM. Posttransplant lymphoproliferative disorder. Chest. 2003;124: 1242-9.

18. Siegel MJ, Lee EY, Sweet SC, Hildebolt C. CT of post-transplantation lymphoproliferative disorder in pediatric recipients of lung allograft. Am J Radiol. 2003;181:1125-31.

19. Hachem RR, Chakinala MM, Yusen RD, Lynch J, Aloush A, Patterson GA, et al. Abdominal-pelvic lymphoproliferative disease after lung transplantation: presentation and outcome. Transplantation. 2004;77: 431-7.

20. Paranjothi S, Yusen RD, Kraus MD, Lynch JP, Patterson GA, Trulock EP. Lymphoproliferative disease after lung transplantation: comparison of presentation and outcome of early and late cases. J Heart Lung Transplant. 2001;20:1054-63.

\section{Discussion}

Dr Shaf Keshavjee (Toronto, Ontario, Canada). Bryan, I congratulate you and your colleagues at Washington University for continuing to lead the field and set the benchmark in lung transplantation. As you have noted, PGD is a particularly devastating complication, and it has a great effect on the cost of this treatment modality. Could you tell us what your definition for PGD in the adult and pediatric population was for the purpose of this study?

Dr Meyers. For the adults, we use the $\mathrm{PaO}_{2} /$ fraction of inspired oxygen ratio in the first 24 hours after transplantation, and the patients who had a ratio of less than 150 were characterized as having PGD. In the pediatric database, if they had radiographic characteristics consistent with reperfusion injury and mechanical ventilation for more than a week or acute retransplantation, they were characterized as having PGD.

Dr Keshavjee. Second, the use of ECMO in children in your experience was about $10 \%$, whereas in adults it was $3 \%$. Is this due to an increased incidence of PGD, or is it perhaps a lower threshold to institute this advanced therapy in a child rather than an adult?

Dr Meyers. Because this study lumps together disparate groups of patients, it is challenging to answer such a question.

As you saw, on the basis of the criteria we used to characterize the patients, the incidence of PGD was the same in adults and children, yet either the severity or the response was different between the pediatric surgeons and the adult surgeons. My sense is that the severity in the children was worse because both groups of surgeons are loathe to start an acute postoperative patient on ECMO, and therefore the profound nature of the hypoxemia in the children seemed to be worse and therefore merit ECMO more frequently.

Dr Keshavjee. Finally, if we are going to encourage the use of living donor transplantation, one of the reasons to do so might theoretically be that you get a better quality graft from a nonbrain-dead donor or shorter ischemic time. Were there any advantages with respect to complications or disadvantages that you noted between your living donor experience and your deceased donor experience?

Dr Meyers. Dr Blackstone would characterize that as an apples and oranges comparison because the living donor recipients were only the pediatric recipients, and they generally all had cystic fibrosis. Our general philosophy has been to primarily use the living donor strategy in patients who would not likely survive to the time it would take to get a cadaveric donor. As a result, that group is negatively biased by worse comorbidities. Therefore I do not know whether such a comparison could be made.

Dr Larry R. Kaiser (Philadelphia, Pa). Bryan, I enjoyed the article very much. I noticed in your most recent time period there seemed to be a slight increase in the incidence of airway complications, as well as a slight increase in the incidence of PGD. Is this because of your efforts to use these worst sort of donors or the marginal sort of donors?

Dr Meyers. We touch on that in the article, and it is disconcerting. You would like to see a gradual shaving away of complications as experience is accumulated, but there certainly have been a lot of changes over the period of time in which these data were accumulated. Use of more marginal donors and donors from longer distance all might contribute to that uptick in the PGD rate and also perhaps the anastomotic complication rate.

We are also liberal in terms of integrating the thoracic surgical fellows and the transplantation fellows into the construction of the anastomosis, and it might be that although the cumulative experience has been gained over 15 years, you reset the clock with each new fellow every 6 months, and therefore there is a constant relearning of what needs to be done.

Dr Malcolm M. DeCamp (Cleveland, Ohio). Bryan, congratulations, that is a fabulous series, and we look forward to you mining these data for years to come to identify that low-hanging fruit.

Over the course of your experience, have you changed any of the procurement techniques, used different preservation solutions, and/or modified your approach to using double-lung grafts, perhaps for more marginal donors? Those things seem more likely to affect PGD rather than some of the other things, such as the use of induction immunosuppression or some of the longer-term determinants of survival. The most dramatic thing that you have shown is that all the factors that affect mortality occur acutely in the hospitalization, whereas the long-term outcomes seem quite similar. I wonder whether you could comment on what you have done differently over the course of time and how those changes might affect your reported outcomes.

Dr Meyers. As you can imagine, there have been a lot of evolutionary changes. We did make a transition from a modification of Euro-Collins to Perfadex a couple of years ago.

We have been very liberal for almost 10 years now using nitric oxide in recipients. We have lately used some inhaled Flolan either instead of or sometimes in addition to nitric oxide at the time of implantation. And the interaction between the quality of the donor and the recipient-there is certainly a real one there, but it is difficult to capture in a talk like this.

Where you would be very reluctant to accept a marginal graft for a single-lung transplant for pulmonary hypertension, you would certainly consider it for a patient with emphysema, and a patient with emphysema has very wide latitude with regard to the size of bilateral lungs that you might put in, whereas other recipients might be more constrained. 
Therefore it is difficult to globalize over the experience, but yes, there are a lot of small evolutionary changes that have taken place.

Dr John Benfield (Los Angeles, Calif). I cannot help but recall coming to Toronto when Joel Cooper had done his first successful lung transplantation and the tribute that he paid at that time to Griff Pearson and to the environment that permitted the development of this field here in Toronto. Environment is so important, and even though this might not add much to the science of what was presented, at least it might add a touch of history.

Dr Thomas L. Spray (Philadelphia, $\mathrm{Pa}$ ). I was quite struck by the fact that the incidence of BOS was the same in the pediatric and adult populations, and yet there have been several other studies from your center suggesting that BOS is less common in the very young patient.

In addition, because very young patients are hard to evaluate for BOS, I wonder whether you could comment on how you made the diagnosis of BOS, especially in the pediatric population, and compared that with the adult population because the diagnosis might be quite difficult to make in a very young patient who cannot do a pulmonary function test.

Dr Meyers. Thanks, Dr Spray, for that question. It is quite a challenge. My recollection of the description from the St Louis Children's Hospital transplantation team about how they measure pulmonary function tests in infants essentially amounts to a forced exhalation measurement with a spirometer on a baby. The child is basically squeezed around the chest and abdomen, and certainly that is a very crude assessment of lung function.

But with regard to the reported freedom of BOS in the youngest children, those children make up a very small fraction of the overall pediatric experience, with a substantial fraction being composed of teenagers who experienced a rate of BOS similar to that observed in the adults. Therefore the classification certainly is not ideal, but this is the best we can go on based on the 2 databases that have been maintained throughout the experience.

\section{Authoritative}

The Journal of Thoracic and Cardiovascular Surgery is the most frequently cited thoracic/cardiovascular surgery journal in the Science Citation Index. An article in JTCVS is cited on average almost twice as often as those in the closest cardiothoracic journal. 


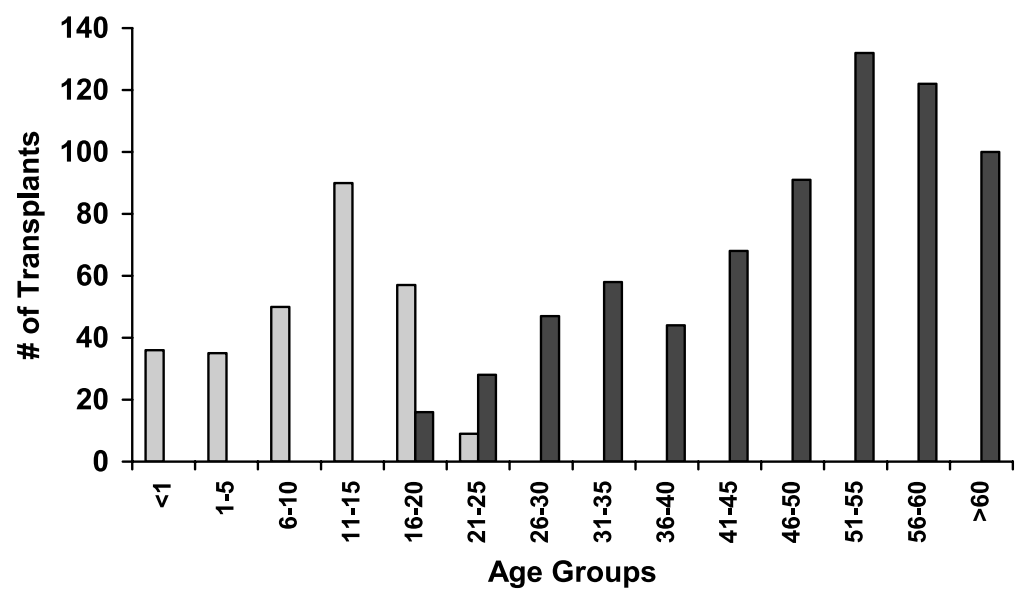

$\square$ Pediatric (n=277) $\square$ Adult $(\mathrm{n}=706)$

Figure E1. Age histogram.

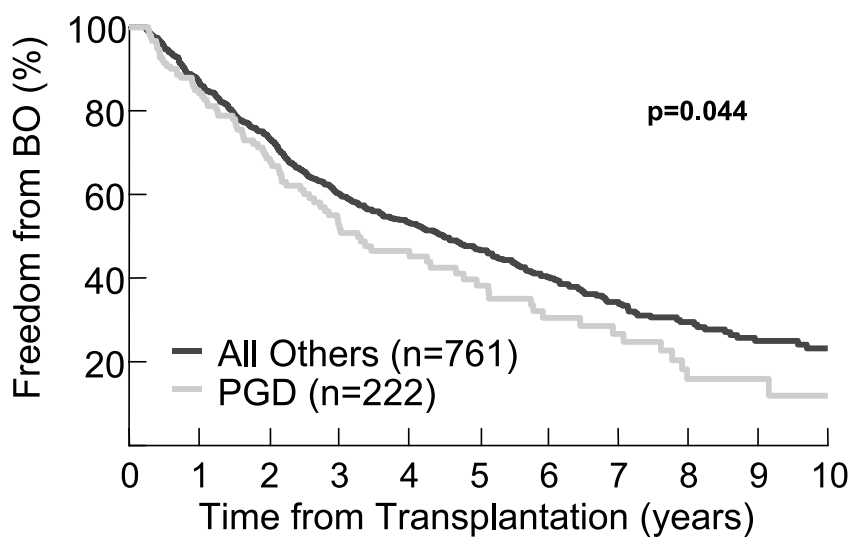

Figure E2. Freedom from bronchiolitis obliterans (BO) after lung transplantation stratified by PGD. 


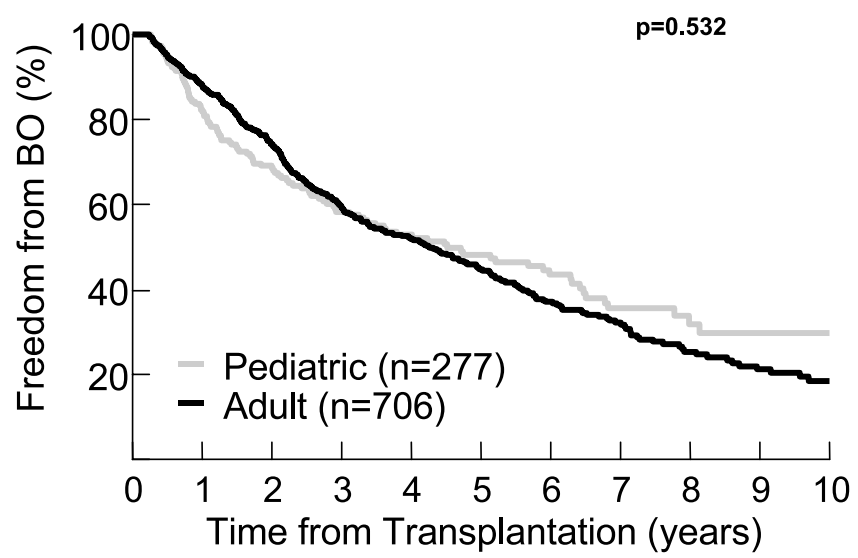

Figure E3. Freedom from bronchiolitis obliterans $(\mathrm{BO})$ after lung transplantation stratified by patient population. 